\title{
Correction: A balance for fairness: fair distribution utilising physics
}

\author{
Song-Ju Kim, Taiki Takahashi \& Kazuo Sano
}

Correction to: Humanities and Social Sciences Communications https://doi.org/10.1057/s41599-021-00806-w, published online 31 May 2021

The affiliation for one of the authors was wrongly stated as:

Song-Ju Kim

SOBIN Institute LLC, Kawanishi, Japan

School of Media and Governance, Keio University, Fujisawa, Japan

It has now been corrected to:

Song-Ju Kim

SOBIN Institute LLC, Kawanishi, Japan

Graduate School of Media and Governance, Keio University, Fujisawa, Japan

This has been corrected in all versions of the paper.

Published online: 24 June 2021

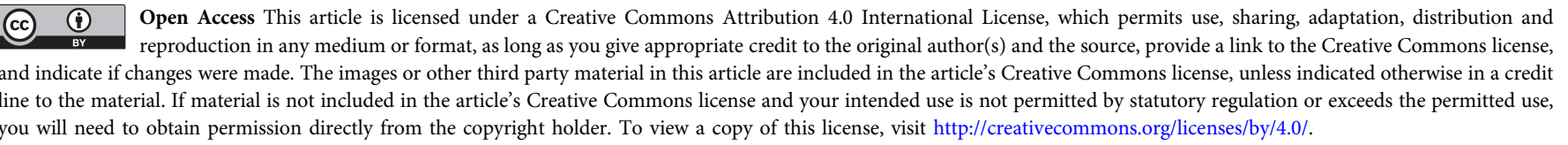

(C) The Author(s) 2021 